\title{
EXTRAÇÃO SEMIAUTOMÁTICA DE CONTORNOS DE TELHADO DE EDIFÍCIOS COM BASE EM SNAKES E PROGRAMAÇÃO DINÂMICA
}

Building roof contour extraction based on snakes and dynamic programming

\author{
ANTONIO JULIANO FAZAN ${ }^{1}$ \\ ALUIR PORFírIO DAL POZ ${ }^{2}$ \\ ${ }^{1}$ Programa de Pós-Graduação em Ciências Cartográficas - FCT/UNESP \\ ${ }^{2}$ Departamento de Cartografia - FCT/UNESP \\ Rua Roberto simonsen, 350 \\ 19.060-900 - Presidente Prudente - SP \\ ajfazan@gmail.com; aluir@fct.unesp.br
}

\section{RESUMO}

Este trabalho apresenta um método para a extração de contornos de telhado de edifícios a partir de imagens digitais tomadas sobre cenas urbanas complexas. $\mathrm{O}$ método proposto é baseado na otimização de uma função de energia snakes, que representa contornos de telhado de edifícios em imagens digitais, através da técnica de otimização por programação dinâmica. Como a grande maioria dos contornos de telhado de edifícios possui lados retilíneos se interceptando em ângulos retos, foram aplicadas restrições à função de energia snakes de modo a atender esta condição geométrica. A principal vantagem de se usar o algoritmo de programação dinâmica para otimizar a função de energia snakes é o aumento do raio de convergência, quando comparado com o que é normalmente obtido na solução original baseada em cálculo variacional. A avaliação experimental foi realizada a partir de dados reais e os resultados obtidos na inspeção visual e análise numérica dos experimentos mostraram o potencial do método para a extração de contornos de telhado de edifícios a partir de imagens digitais.

Palavras-chave: Snakes; Programação Dinâmica; Extração de Edifícios; Análise de Imagens.

\section{ABSTRACT}

This paper presents a method for building roof contours extraction from digital image taken over complex urban scenes. The proposed method is based on the optimization of a snakes' energy function that represents building roof contours in 
digital images by using the dynamic programming optimization technique. As most of the building roof contours contains straight edges intercepting at right angles, appropriate geometrics constraints are enforced into the original snakes' energy function. The main advantage of using the dynamic programming algorithm for optimizing the snakes' energy function is the augmentation of the pull-in-range, when compared to the one that is usually obtained in the original solution based on variational approaches. Experimental evaluation, including visual inspection and numeric analysis, was performed by using real data and the obtained results showed the potentiality of the proposed method for extracting building roof contours from digital imagery.

Keywords: Snake; Dynamic Programming; Building Extraction; Image Analysis.

\section{INTRODUÇÃO}

A extração de feições a partir de imagens digitais tem sido alvo de intensa investigação desde a década de 1970. Este assunto é de grande interesse nas áreas de visão computacional e análise de imagens. No contexto de coleta de dados espaciais para aplicações em mapeamento, novos métodos têm sido desenvolvidos para a extração de feições antrópicas a partir de imagens aéreas e de satélite, tais como edifícios (LEE et al., 2003; LIU et al., 2005; SOHN et al., 2005; LAFARGE et al., 2006; XIONG e ZHANG, 2006; LIU et al., 2008; GUERCKE et al., 2011) e malha viária (GRUEN e LI, 1995; AOURIS et al., 2001; DAL POZ e VALE, 2003; PENG et al., 2005; POULLIS e YOU, 2010; DAL POZ et al., 2010).

Os conceitos de snakes e programação dinâmica (PD) têm sido amplamente explorados em aplicações envolvendo a extração da malha viária, podendo-se citar Gruen e Li (1995), Agouris et al. (2001), Dal Poz e Vale (2003) e Dal Poz et al. (2010). Entretanto, poucas abordagens utilizando snakes têm sido desenvolvidas na área de extração de edifícios. Rüther et al. (2002) utilizaram snakes tradicionais para modelar contornos de edifícios em áreas de assentamentos informais. O método de otimização empregado foi o de PD, cuja inicialização baseava-se na projeção na imagem de contornos aproximados obtidos pela limiarização de um modelo digital de superfície. Guo e Yasuoka (2003) propuseram uma abordagem baseada em snakes para a extração de edifícios a partir da combinação de imagens IKONOS e dados de elevação. Múltiplos indicadores, derivados de ambas as fontes de dados são integrados no modelo snakes de modo a extrair precisamente os contornos de telhado de edifícios. Oriot (2003) apresenta um modelo estatístico de snakes para a extração de edifícios a partir de pares estereoscópicos de imagens aéreas. Esta abordagem separa edifícios de outras regiões a partir da segmentação de uma imagem de disparidades, que é realizada através da busca do polígono que minimiza a energia definida pelo coeficiente de correlação espacial da área de interesse. Baseado no comportamento geométrico e radiométrico dos edifícios, Peng et al. (2005) modificaram o modelo snakes tradicional, a fim de possibilitar uma convergência mais estável para os contornos de edifícios. 
Neste trabalho é proposto um método semiautomático para extrair contornos de telhado de edifícios a partir de imagens digitais. Este método utiliza snakes como base para o desenvolvimento de um modelo matemático representativo desses objetos, cuja solução é obtida através da técnica de otimização por PD. No desenvolvimento do referido modelo é levada em conta a suposição básica de que edifícios se projetam numa imagem como estruturas retilíneas, com seus lados interceptando-se em ângulos aproximadamente retos. A motivação para usar o algoritmo de PD para resolver o problema de otimização baseado em snakes, em vez de usar o método variacional (KASS et al., 1988), vem da possibilidade de se ter um raio de convergência maior, com garantia de convergência. Este artigo está organizado como segue: a Seção 2 apresenta o método proposto. A avaliação experimental e análise dos resultados obtidos é apresentada na Seção 3. Finalmente, as conclusões resultantes da análise dos resultados obtidos nos experimentos realizados são apresentadas na Seção 4.

\section{METODOLOGIA}

A extração de feições a partir de imagens é um procedimento que pode ser adequadamente formulado em termos de um problema de otimização, que por sua vez pode ser solucionado a partir da utilização de técnicas específicas. O requisito básico para tal é a formulação de um modelo matemático representativo do objeto de interesse a ser extraído. Neste trabalho, snakes são usadas como base para a construção de um modelo matemático representando contornos de telhado de edifícios a serem extraídos a partir de imagens digitais. O modelo resultante apresenta uma estrutura particularmente adequada para ser solucionado através do algoritmo de PD. A solução ótima para o modelo são polígonos representando os contornos extraídos de telhado de edifícios.

\subsection{Modelo Matemático de Contornos de Telhado de Edifícios}

Uma snake é descrita em uma imagem por uma curva que se move ao longo das direções $x$ e $y$ sob a influência de forças internas e externas (XU e PRICE, 1998ab). Originalmente foi formulada com base em uma curva paramétrica contínua, mas que, para finalidade de implementação computacional, deve ser substituída por uma curva discreta, dada por uma linha poligonal ou polígono definido por uma sequência de $n$ vértices, conforme mostra a Expressão 1:

$$
v_{i}=\left[\begin{array}{ll}
x_{i} & y_{i}
\end{array}\right], \quad i=0, \ldots, n-1
$$

A função de energia da snakes pode ser expressa na forma dada pela Equação 2 (Kass et al., 1988):

$$
E(v)=\sum_{i=1}^{n-1}\left(\alpha_{i}\left|v_{i+1}-v_{i}\right|^{2}+\beta_{i}\left|v_{i-1}-2 v_{i}+v_{i+1}\right|^{2}\right)+E_{e}(v)
$$

Bol. Ciênc. Geod., sec. Artigos, Curitiba, v. 17, nº 3, p.340-360, jul-set, 2011 
onde: a parte sob o somatório é conhecida como energia interna da snakes; o primeiro e o segundo termos da energia interna são conhecidos como de primeira e segunda ordem, respectivamente; as constantes $\alpha_{i}$ e $\beta_{i}$ são pesos que controlam os termos de primeira e segunda ordens, respectivamente; e $E_{e}(v)$ é conhecido como energia externa.

De uma forma geral, o termo de energia interna possibilita controlar geometricamente a forma da curva $v$ e o termo de energia externa é responsável por mover o contorno $(v)$ em direção à feição de interesse na imagem. Especificamente, a constante $\beta_{i}$ permite controlar a suavidade da curva, sendo que, quanto maior, mais suave será a curva; para $\beta_{i}=0$, a curva $v$ forma uma quina no vértice $v_{i}$. A curva ótima ( $v$ ) é aquela que minimiza a função de energia $E(v)$.

Em se tratando do objeto 'contorno de telhado', a curva $v$ é um polígono que representa o contorno de telhado. Uma característica importante de contornos de telhado de edifícios em imagens digitais é que estes geralmente são delimitados por bordas do tipo degrau, o que permite definir a função de energia externa na forma dada pela Equação 3:

$$
E_{\text {edge }}(v)=\sum_{i=1}^{n-1} \gamma_{i}\left|\nabla G\left(v_{i}\right)\right|^{2}
$$

na qual $\gamma_{i}$ é uma constante negativa e $\left|\nabla G\left(v_{i}\right)\right|$ é a magnitude do gradiente de borda no vértice $i$ do contorno.

Além disso, na maioria dos casos, edifícios são representados por estruturas retilíneas na imagem, definidas por segmentos de borda consecutivos formando ângulos retos em suas quinas ou cantos. Sendo assim, um termo de energia externa adicional pode ser definido através da Equação 4:

$$
E_{\text {corner }}(v)=\sum_{i=0}^{k-1} \eta_{i}\left[\left(1-\cos \left(\delta_{i}\right)\right) \cdot C S\left(v_{i}\right)\right]^{2}
$$

onde $\eta_{i}$ é uma constante negativa, $C S\left(v_{i}\right)$ é a resposta do operador de detecção de quinas e $\delta_{i}$ é o ângulo de incidência das bordas que definem a quina em $v_{i}$. O termo $\left(1-\cos \left(\delta_{i}\right)\right)$ é uma função de ponderação que favorece quinas em ângulos retos.

O modelo matemático de contornos de telhado de edifícios baseado em snakes pode então ser finalmente expresso ao se incorporar as funções de energia externa dadas nas Equações 3 e 4 na Equação 2, resultando na Equação 5: 


$$
E_{2}(v)=\sum_{i=0}^{n-1}\left[\begin{array}{l}
\alpha_{i}\left|v_{i+1}-v_{i}\right|^{2}+\beta_{i}\left|v_{i-1}-2 v_{i}+v_{i+1}\right|^{2} \\
-\gamma_{i}\left|\nabla G\left(v_{i}\right)\right|^{2}-\eta_{i}\left[\left(1-\cos \left(\delta_{i}\right)\right) C S\left(v_{i}\right)\right]^{2}
\end{array}\right] .
$$

Os valores dos pesos $\beta_{i}, \gamma_{i}$ e $\eta_{i}$ dependem do tipo de descontinuidade existente no vértice $v_{i}$ : borda degrau ou quina. Todos os pesos (incluindo $\alpha_{i}$ ) são positivos, mas em se tratando de uma quina, tem que haver em $v_{i}$ uma mudança abrupta na direção do polígono. Isto implica na necessidade de anular $\beta_{i}$ para possibilitar uma descontinuidade de segunda ordem no vértice $v_{i}$. Além disso, o termo de energia de borda (Equação 3) passa a não ter poder de discriminação da descontinuidade em $v_{i}$, implicando na necessidade de se ter $\gamma_{i}=0$. Ao contrário, quando em $v_{i}$ estiver presente uma descontinuidade do tipo borda degrau, o termo de energia de quina (Equação 4) não terá poder discriminatório. Nesse caso, deve-se anular o peso $\eta_{i}$.

\subsection{Otimização do Modelo de Contornos de Telhado de Edifícios Através de PD}

Se as variáveis de uma função de energia num problema de otimização não estiverem simultaneamente inter-relacionadas, então uma maneira eficiente para obter a solução deste problema é através da técnica de PD (BALLARD e BROWN, 1982). Seja o problema de encontrar o conjunto de valores ótimos $\left(\bar{x}_{1}, \ldots, \bar{x}_{n}\right)$ que minimizam a função de energia $g=g\left(x_{1}, \ldots, x_{n}\right)$. Se a função de energia $g$ puder ser escrita como uma soma de subfunções dependentes de subconjuntos de $\left(x_{1}, \ldots, x_{k}\right)$, com $k \square n$, como a dada pela Equação 6:

$$
\begin{aligned}
g & =g\left(x_{1}, \ldots, x_{n}\right)=g_{1}\left(x_{1}, x_{2}, x_{3}\right)+\cdots+g_{n-2}\left(x_{n-2}, x_{n-1}, x_{n}\right) \\
& =\sum_{i=1}^{n-2} g_{i}\left(x_{i}, x_{i+1}, x_{i+2}\right)
\end{aligned}
$$

então um procedimento de múltiplos estágios como o algoritmo de PD pode ser aplicado. Maiores detalhes a respeito deste algoritmo podem ser encontrados em Ballard e Brown (1982) e Gruen e Li (1995).

Uma simples análise da equação de energia dada pela Equação 5 mostra que apenas três vértices consecutivos $\left(v_{i-1}, v_{i}, v_{i+1}\right)$ da linha poligonal $v$ estão simultaneamente inter-relacionados e, desta forma, a mesma pode ser decomposta 
em uma soma de $n-1$ subfunções $E_{i}\left(v_{i-1}, v_{i}, v_{i+1}\right)$, permitindo escrever a Equação 7:

$$
E_{2}(v)=\sum_{i=0}^{n-1} E_{i}\left(v_{i-1}, v_{i}, v_{i+1}\right)
$$

A estrutura da Equação 7 se assemelha à estrutura da Equação 6 e, consequentemente, o algoritmo de otimização por PD pode ser eficientemente aplicado na otimização da função de energia dada na Equação 5. Vale notar neste caso que seis variáveis (duas para cada vértice) se inter-relacionam simultaneamente.

De modo a inicializar o processo de extração, alguns pontos sementes descrevendo uma aproximação para o contorno a ser extraído devem ser fornecidos na imagem. De uma forma geral, os pontos sementes devem ser posicionados nas vizinhanças das quinas do contorno de telhado. Não é necessário colocar pontos sementes ao longo dos lados do contorno, isto porque estes podem ser preditos levando em conta que estes lados são retilíneos.

O polígono inicial fornecido no início do processo de extração é descrito por segmentos retos definidos por pares de pontos sementes consecutivos, conforme ilustrado pela Figura 1.

Figura 1 - Contorno inicial aproximado utilizado na inicialização do processo de extração.

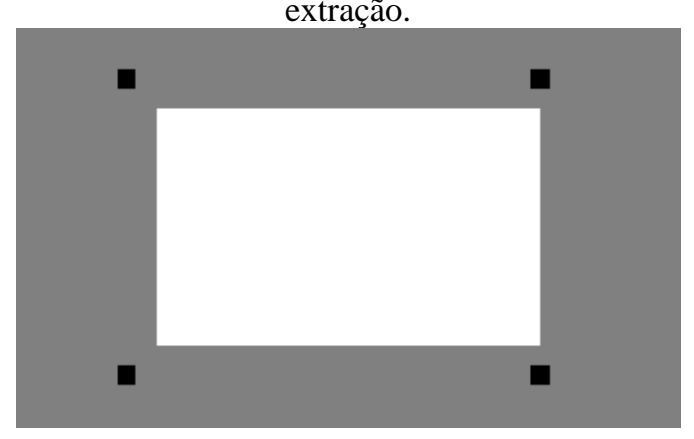

A Figura 2 mostra como o espaço de busca é construído. Têm-se dois tipos de pontos que geram os polígonos candidatos à solução ótima (polígono ótimo): pontos representativos dos lados do contorno e correspondentes à descontinuidade do tipo borda degrau; e pontos representativos das quinas e correspondentes à descontinuidade do tipo quina. A princípio, parece simples determinar as quinas e uni-las para formar o polígono do contorno de telhado. Entretanto, podem ser detectadas falsas quinas e até mesmo não existirem quinas para um dado canto do 
contorno de telhado. Daí a importância de incluir pontos de lados de contorno para prover suporte à determinação de quais são as quinas corretas.

Figura 2 - Amostragem do espaço de busca.

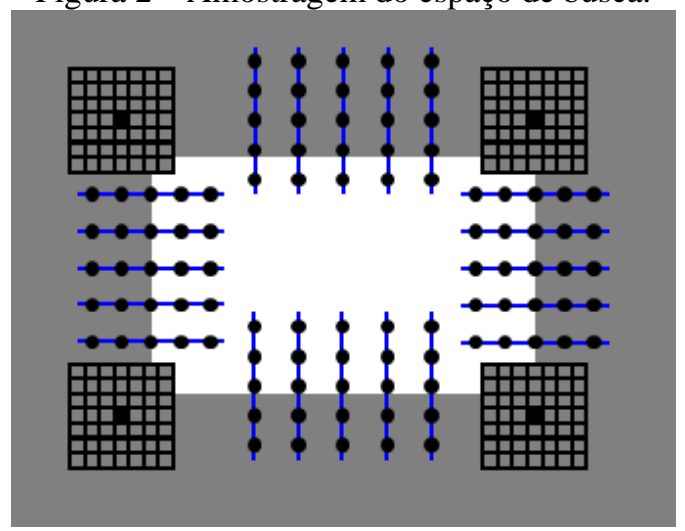

Quanto aos pontos candidatos para a representação de um canto de edifício, um ponto de referência deve ser fornecido pelo operador, sendo os demais determinados por um algoritmo de detecção de quinas sobre uma pequena subimagem delimitada em torno do respectivo ponto semente (Figura 2). As dimensões dessa subimagem devem ser suficientes para que a mesma contenha a quina correta do edifício. O detector de quinas utilizado é o de Harris (HARRIS e STEPHENS, 1988) e as quinas com melhor resposta são armazenadas, juntamente com os ângulos $\left(\delta_{i}\right)$ de incidência das bordas nas respectivas quinas. $\mathrm{O}$ armazenamento de múltiplas quinas é necessário, pois existe a possibilidade de a quina com melhor resposta dentro da janela de busca ser uma falsa positiva. Quanto aos pontos candidatos para a representação de pontos de lados de contorno de edifício, os mesmos são amostrados regularmente ao longo de seções transversais aos lados de contornos de edifício definidos por pares de pontos sementes (Figura 2). É importante notar que as seções transversais também são amostradas regularmente ao longo dos lados de contorno de edifício. Como são centradas em pontos dos lados do contorno definido pelos pontos sementes (polígono inicial), não existe preferência de busca em um ou outro lado.

Para se ter uma ideia do tamanho do espaço de busca, supor que cada quina de edifício possua $m$ candidatas. Supor também que em cada seção transversal aos lados de contorno de edifício sejam amostrados $m$ pontos candidatos. Sendo $n$ a soma entre o número de pontos sementes e o número total de seções transversais amostradas, existirão no espaço de busca $m^{n}$ polígonos. O polígono ótimo é aquele que minimiza a função de energia dada pela Equação 5.

A solução do problema de otimização por PD é encontrada em duas etapas: 
1) Refinamento do polígono inicial definido pelos pontos sementes: a estratégia descrita acima é aplicada para refinar o polígono inicial (definido pelos pontos sementes) fornecido no início do processo de extração. A resolução típica (distância entre pontos amostrados) adotada para as seções transversais é de um pixel. Já o número de elementos nestas seções deve ser compatível com a qualidade do polígono inicial. No exemplo ilustrativo da Figura 3, os pontos em vermelho correspondem ao conjunto de vértices obtidos por otimização.

Figura 3 - Otimização do contorno inicial por PD.

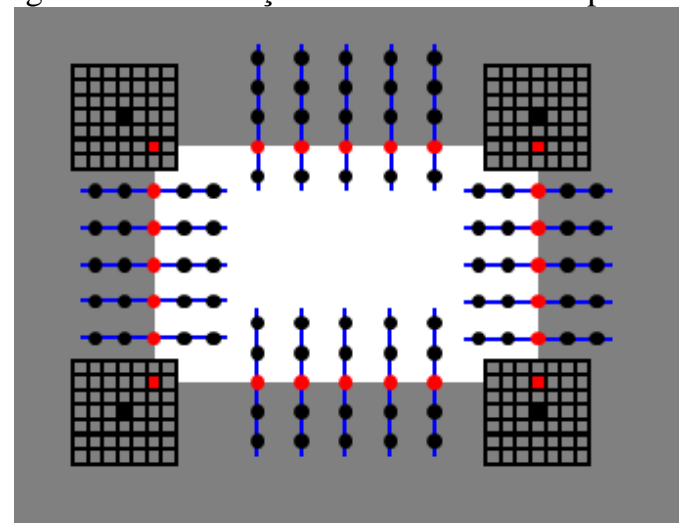

2) Refinamento da primeira solução: como os pontos obtidos ao longo dos lados de contorno podem estar afetados por anomalias locais (por exemplo, um pequeno detalhe do edifício), provocando irregularidades locais no contorno, um método robusto de regressão pode ser utilizado para obter retas que melhor modelam os lados de contorno de edifício. A seguinte estratégia é utilizada: 1) determina-se por mínimos quadrados as equações das retas que representam lados de contornos; 2) eliminam-se para cada reta os pontos que distam mais que o desvio-padrão $(\sigma)$ das distâncias dos pontos à reta considerada; 3) recalculam-se as regressões lineares usando os respectivos conjuntos de pontos remanescentes. Na sequência, com base nestas retas, determina-se por intersecção as novas quinas, as quais farão o papel de pontos sementes para uma nova otimização da equação de energia (Equação 5). Uma nova otimização é indicada pelos seguintes motivos (Figura 4): as retas determinadas por regressão podem ainda estar afetadas por anomalias existentes, não modeladas, ao longo dos lados de contorno; as seções transversais de busca com resolução bem melhor (0,5 pixel) são adotadas para melhorar a qualidade final das retas representando lados de contorno, melhorando também as posições finais dos vértices de quina do polígono de telhado; os comprimentos das seções transversais de busca e as 
dimensões das subimagens para as novas quinas são fixadas iguais ao desvio-padrão $(\sigma)$ determinado acima, tornando a solução de otimização menos sensível às anomalias existentes nas adjacências do contorno de telhado. Após o novo processo de otimização, determinam-se novamente as retas por regressão robusta e, a partir destas, as posições finais das quinas dos edifícios. O polígono final de contorno de telhado é obtido em função destas quinas (Figura 5).

Figura 4 - Refinamento da solução com restrição do espaço de busca.

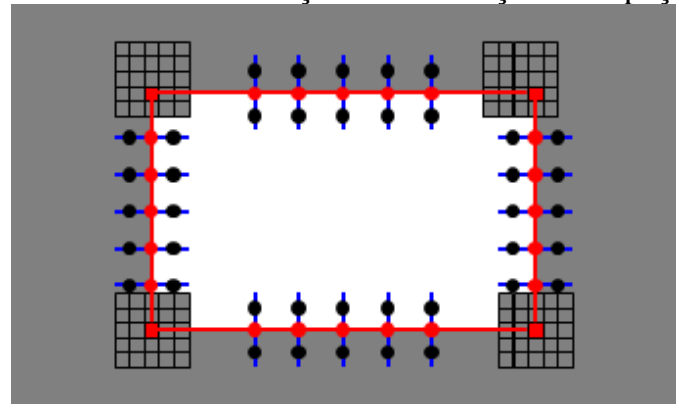

Figura 5 - Contorno final extraído para o telhado de edifício.

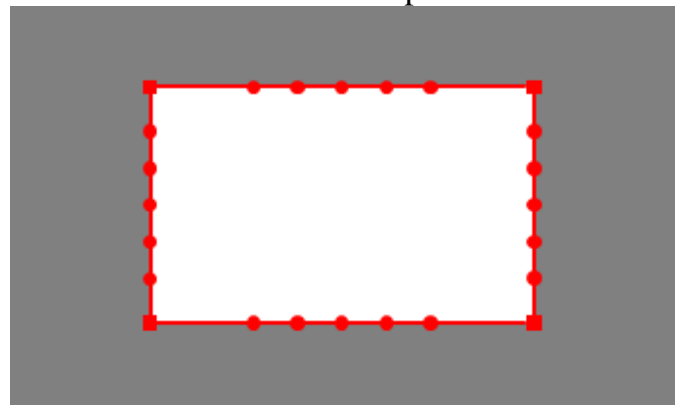

\section{AVALIAÇÃO EXPERIMENTAL}

De modo a avaliar o método desenvolvido, foram realizados vários experimentos utilizando dados reais. Na sequência, três destes experimentos são descritos. Além da inspeção visual dos resultados obtidos, uma análise numérica foi realizada para cada experimento. Esta avaliação é baseada na estimação de parâmetros numéricos de qualidade, a partir da comparação dos contornos extraídos via algoritmo computacional com os contornos correspondentes extraídos manualmente pelo operador e utilizados como referência. Para os experimentos realizados, foram calculados valores de completeza de área $\left(C_{A}\right)$ e $R M S E$ (root mean square error). A completeza de área é estimada a partir das áreas $A_{E}$ e $A_{R}$ 
dos polígonos que descrevem um contorno extraído e seu respectivo contorno de referencia. Esta medida é dada pela Equação 8 (Rüther et al., 2002):

$$
C_{A}=\left(1-\frac{\left|A_{E}-A_{R}\right|}{A_{R}}\right) \cdot 100 \%
$$

A completeza de área é uma medida percentual da área $A_{E}$ do objeto extraído, comparada à área $A_{R}$ do objeto de referência. O parâmetro $C_{A}$ tem por suposição básica a existência de apenas discrepâncias locais entre o contorno extraído e o respectivo contorno de referência, isto é, ambos os contornos não devem apresentar discrepâncias globais, como as que resultam de transformação de escala, rotação ou translação. Como a suposição acima é cumprida pelo método proposto, as discrepâncias entre ambos os contornos podem ser avaliadas em função de discrepâncias das áreas entre eles.

O RMSE é calculado em função das distâncias entre os vértices descrevendo um contorno extraído e seu respectivo contorno de referência. Este parâmetro fornece uma estimativa da distância média entre tais vértices e é calculado a partir da Equação 9:

$$
R M S E=\sqrt{\frac{1}{n} \sum_{i=1}^{n} d_{i}^{2}}
$$

na qual $d_{i}$ é a distância entre o vértice $i$ do contorno de referência e o seu correspondente no contorno extraído e $n$ é o número total de vértices do contorno extraído.

Os testes realizados permitiram verificar que o termo de primeira ordem da função de energia (Equação 5) não auxiliava na discriminação de pontos de borda pertencentes ao contorno de telhado de um edifício. Sendo assim, em todos os experimentos o parâmetro $\alpha_{i}$ foi definido como zero em todos os vértices $v_{i}$ da curva representando um contorno de telhado de edifício.

\subsection{Experimento 1}

Nesta seção são apresentados os resultados obtidos no primeiro experimento realizado. O principal objetivo deste experimento foi avaliar o desempenho do método na tarefa de extrair o mesmo contorno de telhado a partir de diferentes aproximações iniciais, permitindo assim verificar a repetibilidade dos resultados, o que é um indicativo de robustez do método. Foi escolhido um edifício cujos limites (bordas) estão bem definidos na imagem. Ao todo foram realizadas quatro extrações do mesmo contorno de telhado, a partir dos contornos aproximados apresentados nas Figuras 6(a), 6(b), 6(c) e 6(d), respectivamente. 
Figura 6 - Experimento 1: Contornos aproximados utilizados na inicialização do método de extração.

(a)

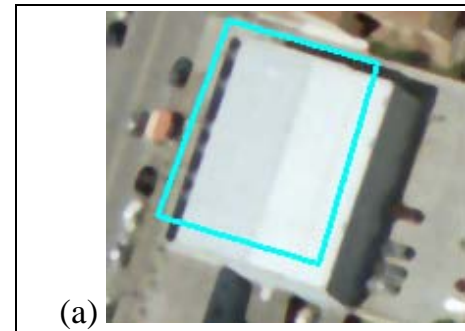

(c)

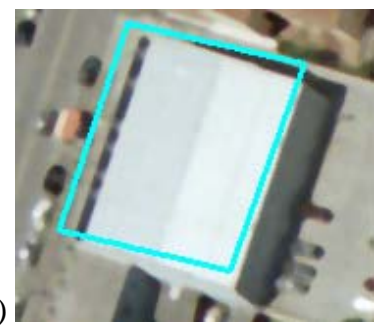

(b)

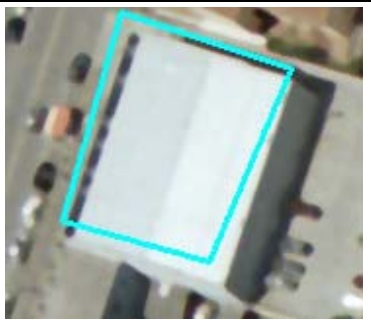

(d)

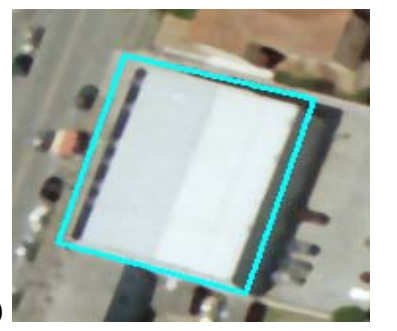

Os correspondentes contornos extraídos pelo algoritmo computacional são apresentados nas Figuras 7(a), 7(b), 7(c) e 7(d), respectivamente.

Figura 7 - Experimento 1: Contornos extraídos pelo algoritmo computacional do método de extração.

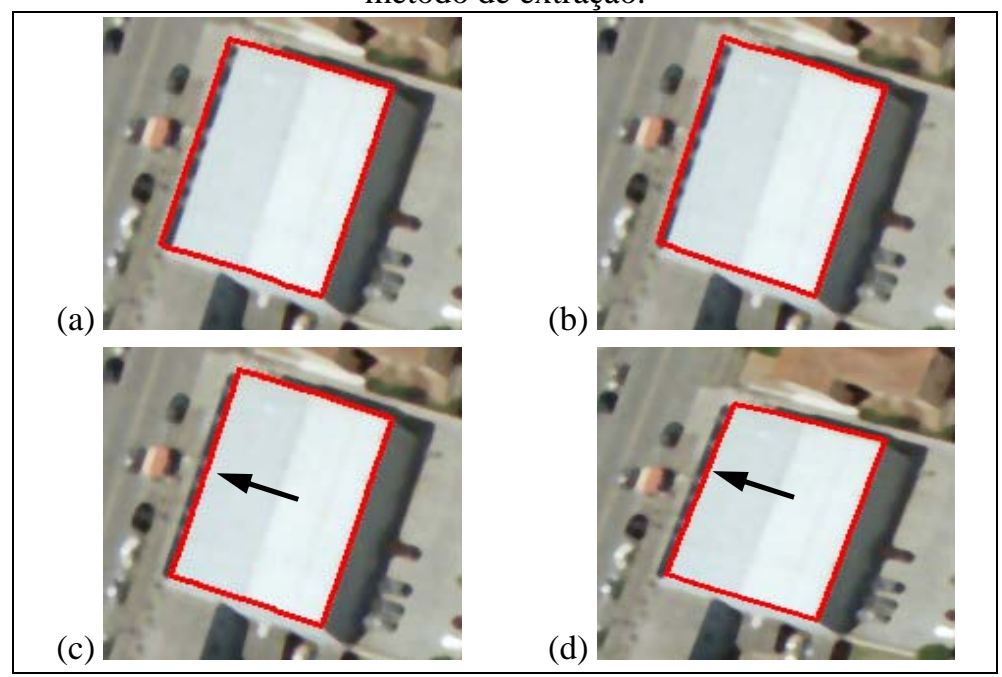

Bol. Ciênc. Geod., sec. Artigos, Curitiba, v. 17, nº 3, p.340-360, jul-set, 2011 
A partir de uma inspeção visual dos resultados obtidos, é possível verificar que o método de extração foi bem sucedido em todos os casos. Entretanto, nos casos dos resultados mostrados nas Figuras 7(c) e 7(d), um dos segmentos do contorno (em destaque nas respectivas figuras) foi incorretamente extraído, devido à ocorrência de uma projeção de sombra vizinha ao limite real do telhado. Em alguns casos, as regiões de transição entre sombras e objetos vizinhos podem resultar em fortes respostas a um detector de bordas e, quando este fenômeno ocorre próximo a um contorno de telhado, pode interferir negativamente no processo de extração. De qualquer maneira, sem considerar os efeitos negativos da interação entre sombras e edifícios, é possível afirmar que o método apresenta boa repetibilidade na extração de contornos a partir de diferentes aproximações iniciais.

A análise numérica foi realizada a partir da comparação de cada um dos contornos extraídos com o contorno de referência apresentado na Figura 8. Para efeito de estimação dos parâmetros numéricos de qualidade, foram consideradas as quinas que descrevem ambos os contornos (extraído e de referência, respectivamente). Os valores resultantes são apresentados na Tabela 1.

Figura 8 - Contorno de referência utilizado na análise numérica dos resultados obtidos no Experimento 1.

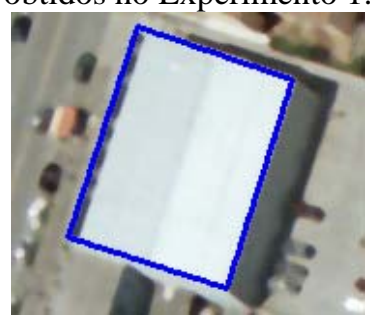

Tabela 1: Parâmetros estimados na análise numérica do Experimento 1.

\begin{tabular}{c|c|c|c|c}
\hline \multirow{2}{*}{ Parâmetros } & \multicolumn{4}{|c}{ Contorno } \\
\cline { 2 - 5 } & $\mathbf{1}$ & $\mathbf{2}$ & $\mathbf{3}$ & $\mathbf{4}$ \\
\hline$C_{A}$ (\%) & 97,8 & 98,8 & 95,8 & 96,1 \\
\hline$R M S E$ (pixels) & 1,580 & 1,904 & 3,711 & 4,198 \\
\hline
\end{tabular}

Os resultados obtidos na análise numérica indicam que os contornos extraídos possuem boa correspondência com os limites reais dos telhados presentes na imagem. De um modo geral, o método de extração funcionou adequadamente, mesmo nos casos dos contornos afetados pelas projeções de sombra, o que causou piora nos valores de $C_{A}$ e RMSE. 


\subsection{Experimento 2}

No segundo experimento realizado, foram utilizados dois segmentos de uma imagem contendo diversos edifícios em cada uma das regiões correspondentes a estes segmentos. No caso deste experimento, seis dos contornos de telhado presentes nos segmentos de imagem foram extraídos. As Figuras 9(a) e 9(b) ilustram os contornos utilizados como aproximação inicial para o método de extração.

Figura 9 - Experimento 2: Contornos aproximados utilizados na inicialização do método de extração.

(a)

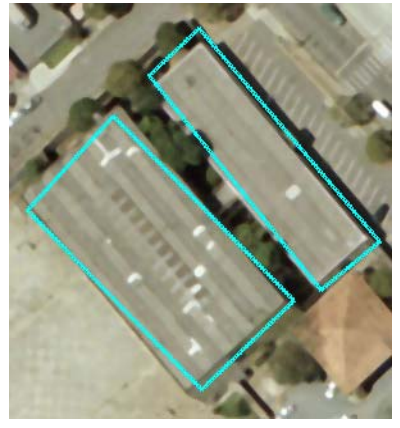

(b)

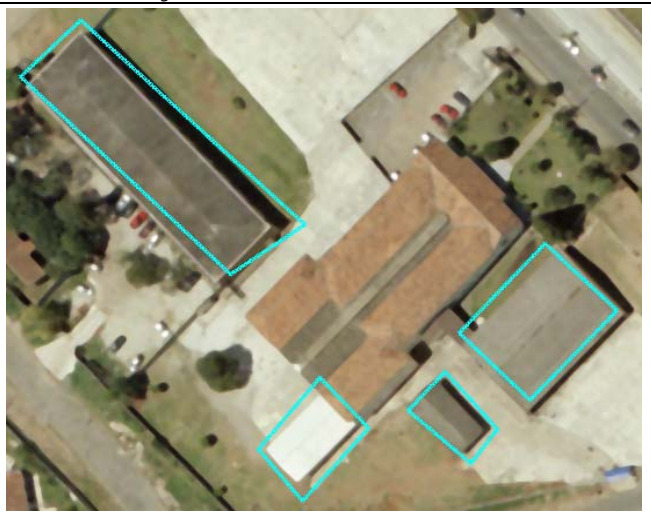

Os correspondentes contornos extraídos são apresentados nas Figuras 10(a) e 10(b), respectivamente.

Figura 10 - Experimento 2: Contornos extraídos pelo algoritmo computacional do método de extração.

(a)

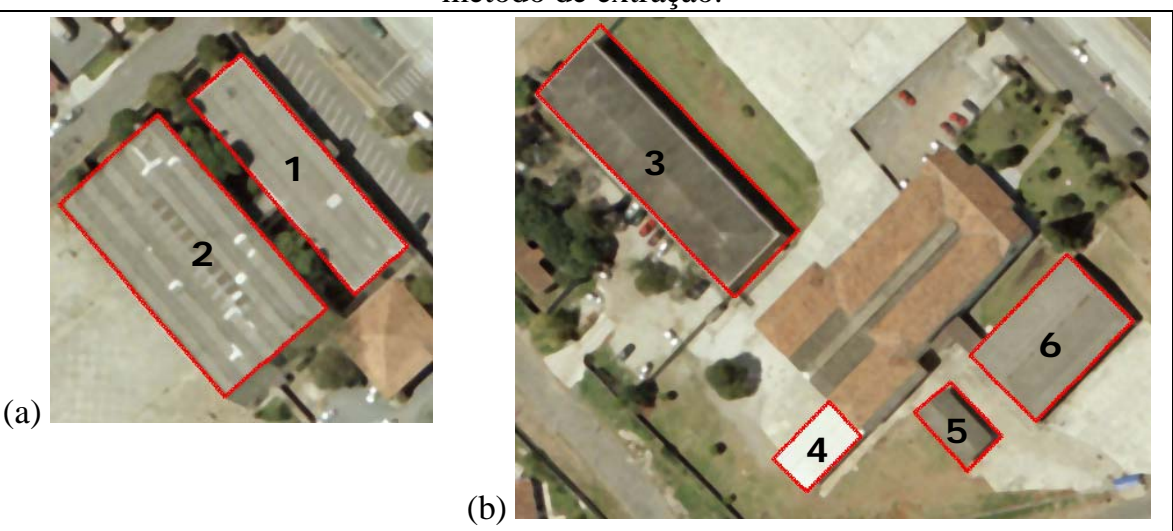

Bol. Ciênc. Geod., sec. Artigos, Curitiba, v. 17, nº 3, p.340-360, jul-set, 2011 
Por fim, as Figuras 11(a) e 11(b) ilustram os contornos de referência utilizados na análise numérica dos resultados obtidos neste experimento.

Figura 11 - Experimento 2: Contornos de referência utilizados na análise numérica dos resultados.

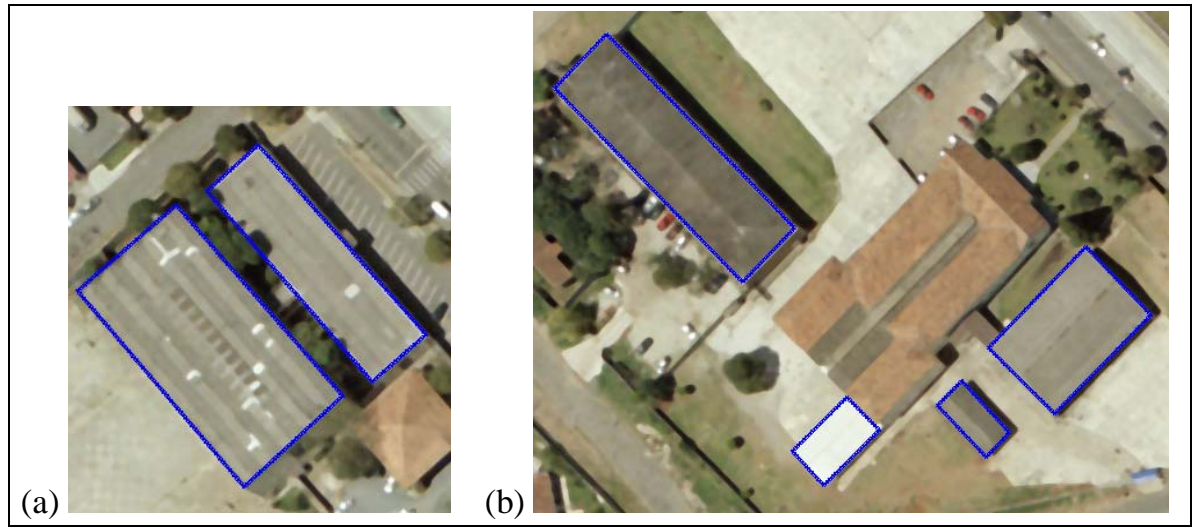

A inspeção visual dos resultados obtidos indica que o método de extração foi bem sucedido nos casos dos contornos 1, 2, 4 e 6 . No caso dos demais contornos (3 e 5), a presença de sombras projetadas pelos próprios edifícios influenciou negativamente os resultados obtidos na extração. No caso destes contornos, dois de seus segmentos foram confundidos com a borda resultante das projeções de sombra.

De fato, em alguns casos o método não funciona adequadamente por causa da influência de objetos vizinhos como árvores próximas aos telhados e projeção de sombras, conforme já foi comentado no experimento 1. Quando uma borda provocada pela projeção de sombra de um telhado apresenta maiores respostas a um detector que a borda correspondente ao contorno do próprio telhado, este pode ser incorretamente extraído nesta região. Isto também ocorre porque, nestes casos, a borda correspondente ao contorno de telhado e a resultante da projeção de sombra do telhado são aproximadamente paralelas, o que significa que tanto pontos de borda amostrados sobre o contorno real quanto pontos amostrados sobre bordas de sombras apresentam o mesmo padrão de alinhamento, descrevendo linhas poligonais formadas por segmentos colineares. A implicação deste fato é que, nestes casos, a função de energia interna do modelo dado pela Equação 5 não auxilia na discriminação dos pontos de borda pertencentes ao contorno, uma vez que o valor resultante da função de energia externa predomina na energia total correspondente àqueles pontos de borda. Consequentemente, o algoritmo de $\mathrm{PD}$ seleciona os pontos que resultam no menor valor global de energia. A Figura 12 ilustra um exemplo desta situação, correspondente ao segmento do contorno 3 que foi incorretamente extraído neste experimento. 
Figura 12 - Exemplo ilustrativo da influência de bordas resultantes da projeção de sombras sobre os resultados obtidos pelo processo de extração.

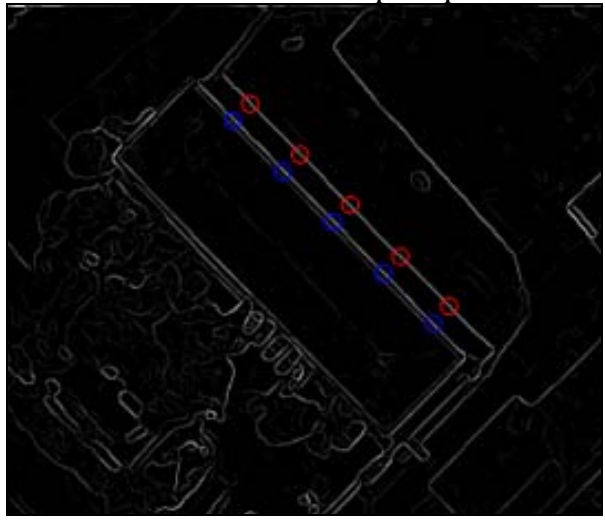

A região do mapa de bordas mostrada na referida figura apresenta pontos amostrados ao longo da borda correspondente ao segmento real do contorno (destacados em azul), bem como pontos amostrados ao longo da borda resultante da projeção da sombra do edifício (destacados em vermelho). Neste caso, a energia resultante nestes pontos foi menor que a dos pontos reais do contorno, causando seu deslocamento para a borda resultante da projeção de sombra presente nesta região, conforme pode ser visto através da inspeção visual da Figura 10(b).

Os parâmetros estimados na avaliação numérica dos resultados são apresentados na Tabela 2. Os valores obtidos comprovam as observações resultantes da inspeção visual dos contornos extraídos. Os contornos 1, 2, 4 e 6 resultaram em altos valores de $C_{A}$ e baixos valores de RMSE, o que significa boa correspondência entre os contornos extraídos pelo algoritmo e os contornos reais presentes na imagem. Nos demais casos (contornos 3 e 5), os valores de $C_{A}$ são menores e os de RMSE maiores, o que indica menor correspondência entre os contornos extraídos e os correspondentes presentes na imagem. O pior resultado ocorreu no caso do contorno 5, indicando falha no processo de extração.

Tabela 2: Parâmetros estimados na análise numérica do Experimento 2.

\begin{tabular}{c|c|c|c|c|c|c}
\hline \multirow{2}{*}{ Parâmetro } & \multicolumn{7}{c}{ Contorno } \\
\cline { 2 - 7 } & $\mathbf{1}$ & $\mathbf{2}$ & $\mathbf{3}$ & $\mathbf{4}$ & $\mathbf{5}$ & $\mathbf{6}$ \\
\hline$C_{A}$ (\%) & 98,4 & 98,6 & 69,6 & 97,2 & 41,9 & 99,9 \\
\hline$R M S E$ (pixels) & 1,397 & 2,079 & 13,893 & 1,121 & 51,675 & 1,524 \\
\hline
\end{tabular}




\subsection{Experimento 3}

A Figura 13 ilustra os resultados do terceiro experimento realizado. Neste caso foi selecionado um segmento de imagem contendo alguns edifícios e realizada a extração de alguns de seus contornos de telhado. A Figura 13(a) ilustra os contornos iniciais utilizados como aproximação para o método e os correspondentes contornos extraídos são mostrados na Figura 13(b). Os contornos de referência utilizados na análise numérica dos resultados são ilustrados pela Figura 13(c). Neste experimento ocorreram problemas de extração com os contornos 1, 2 e 6 . É importante notar também que os lados curvos do contorno 4 são grosseiramente aproximados pelo método proposto, o que é previsível, visto que o mesmo foi desenvolvido para tratar contornos de telhados definidos por segmentos retilíneos.

Figura 13 - Experimento 3: (a) Contornos iniciais aproximados; (b) Contornos extraídos via método computacional; (c) Contornos de referência utilizados na avaliação numérica dos resultados.

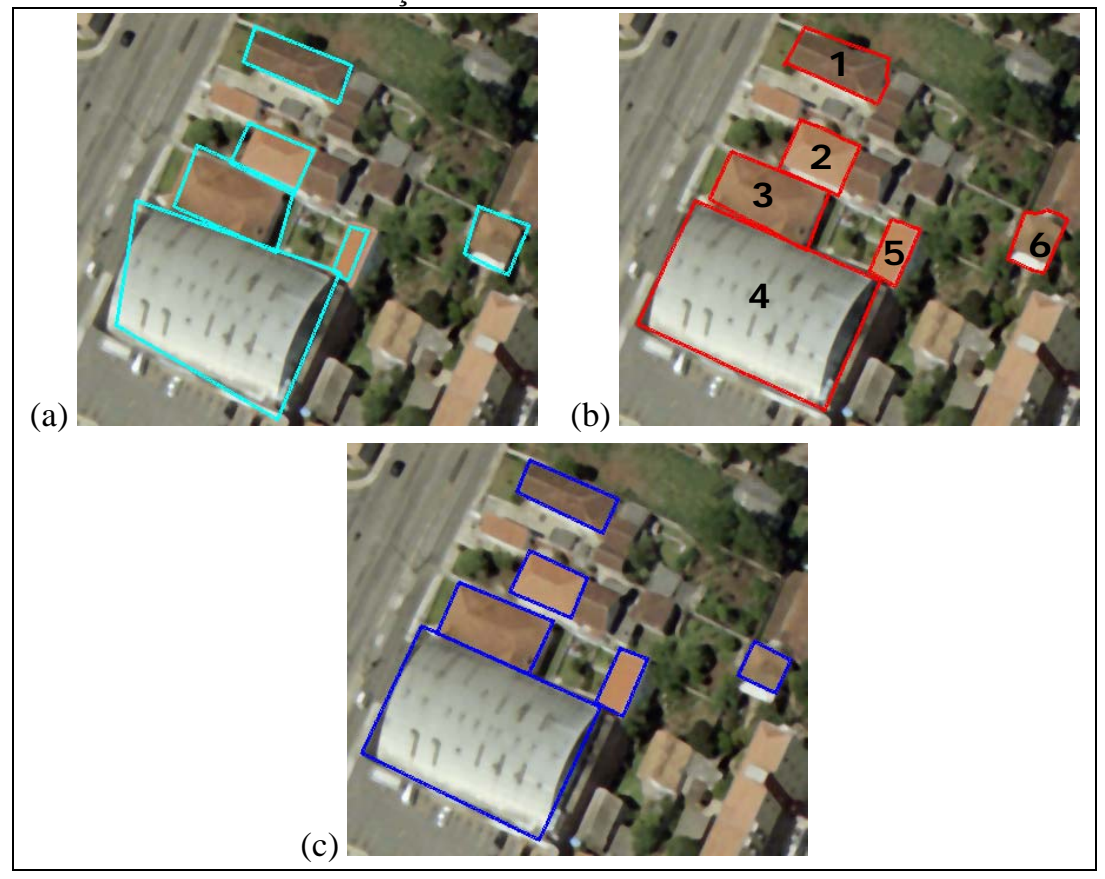

A Tabela 3 apresenta os valores dos parâmetros de qualidade estimados na análise numérica dos resultados obtidos neste experimento. De um modo geral, os valores indicam que a extração dos contornos 3, 4 e 5 foi bem sucedida. Conforme foi dito, a extração do contorno 2 apresentou problemas, pois um dos segmentos que o define foi incorretamente extraído, em razão de o mesmo ter sido atraído para uma 
borda vizinha ao telhado de maior energia. Esta borda não é resultante da projeção de uma sombra, mas trata-se de uma borda verdadeira correspondente a uma região de transição entre objetos e que apresentou maiores respostas ao detector de bordas utilizado. A partir deste exemplo, é possível verificar que a interação entre objetos vizinhos pode trazer efeitos negativos ao processo de extração e produzir resultados incorretos, como no caso apresentado. Uma possibilidade para contornar este problema seria restringir o espaço de busca do método durante a amostragem dos pontos de borda, mas em alguns casos isto também poderia trazer efeitos negativos como a não convergência para o contorno real na imagem devido à amostragem insuficiente de pontos de borda. Os demais resultados (contornos 1 e 6) apresentam indicadores de qualidade compatíveis com as falhas ocorridas, destacando-se o baixo valor de $C_{A}$ obtido para o contorno 6 .

Tabela 3: Parâmetros estimados na análise numérica do Experimento 3.

\begin{tabular}{c|c|c|c|c|c|c}
\hline \multirow{2}{*}{ Parâmetro } & \multicolumn{7}{|c}{ Contorno } \\
\cline { 2 - 7 } & $\mathbf{1}$ & $\mathbf{2}$ & $\mathbf{3}$ & $\mathbf{4}$ & $\mathbf{5}$ & $\mathbf{6}$ \\
\hline$C_{A}$ (\%) & 78,8 & 77,4 & 91,3 & 99,8 & 98,9 & 51,4 \\
\hline$R M S E$ (pixels) & 4,533 & 5,297 & 4,460 & 7,393 & 1,172 & 8,628 \\
\hline
\end{tabular}

Além do problema da projeção de sombras vizinhas ao telhado descrito no experimento anterior, outro fator que limita o desempenho do método de extração é a área compreendida por um telhado na imagem. Este fator interfere no processo de amostragem dos vértices que descrevem seu contorno, uma vez que em alguns casos de áreas pequenas o espaço de busca pode se tornar muito restrito, impossibilitando a amostragem e seleção de um número suficiente de vértices para descrição e complementação (definição dos vértices de quina) do contorno. A ocorrência simultânea deste fenômeno com a presença de bordas mal definidas na região do contorno faz com que o processo de extração falhe nestas ocasiões.

A Figura 14 destaca algumas regiões do mapa de bordas gerado na extração dos contornos de telhado obtidos no experimento 3 . O detalhe 1 destaca a região do mapa de bordas correspondente ao contorno 1 extraído neste experimento. Este exemplo ilustra o caso de bordas mal definidas na imagem, que interferem negativamente no processo de extração, produzindo resultados incorretos como o que foi obtido para o contorno 1 . Já o detalhe 2 destaca a região do mapa de bordas correspondente ao contorno 6. Embora neste caso as bordas do contorno estejam bem definidas, o edifício apresenta pequena área na imagem, inviabilizando a amostragem de um número adequado de pontos representativos do contorno de telhado. Além disso, existe a influência negativa de uma borda de resposta mais elevada na parte inferior desta região do mapa. A combinação destes fatores provocou a falha do processo de extração neste caso. Por fim, o detalhe 3 destaca a

Bol. Ciênc. Geod., sec. Artigos, Curitiba, v. 17, nº 3, p.340-360, jul-set, 2011 
região correspondente ao contorno 3, que foi corretamente extraído embora suas bordas apresentem baixa resposta em algumas partes da região do contorno. Este fato demonstra que o método de extração é relativamente robusto e funciona corretamente mesmo em algumas situações desfavoráveis como a exemplificada neste caso.

Figura 14 - Influência de bordas mal definidas e edifícios de pequenas áreas no processo de extração.

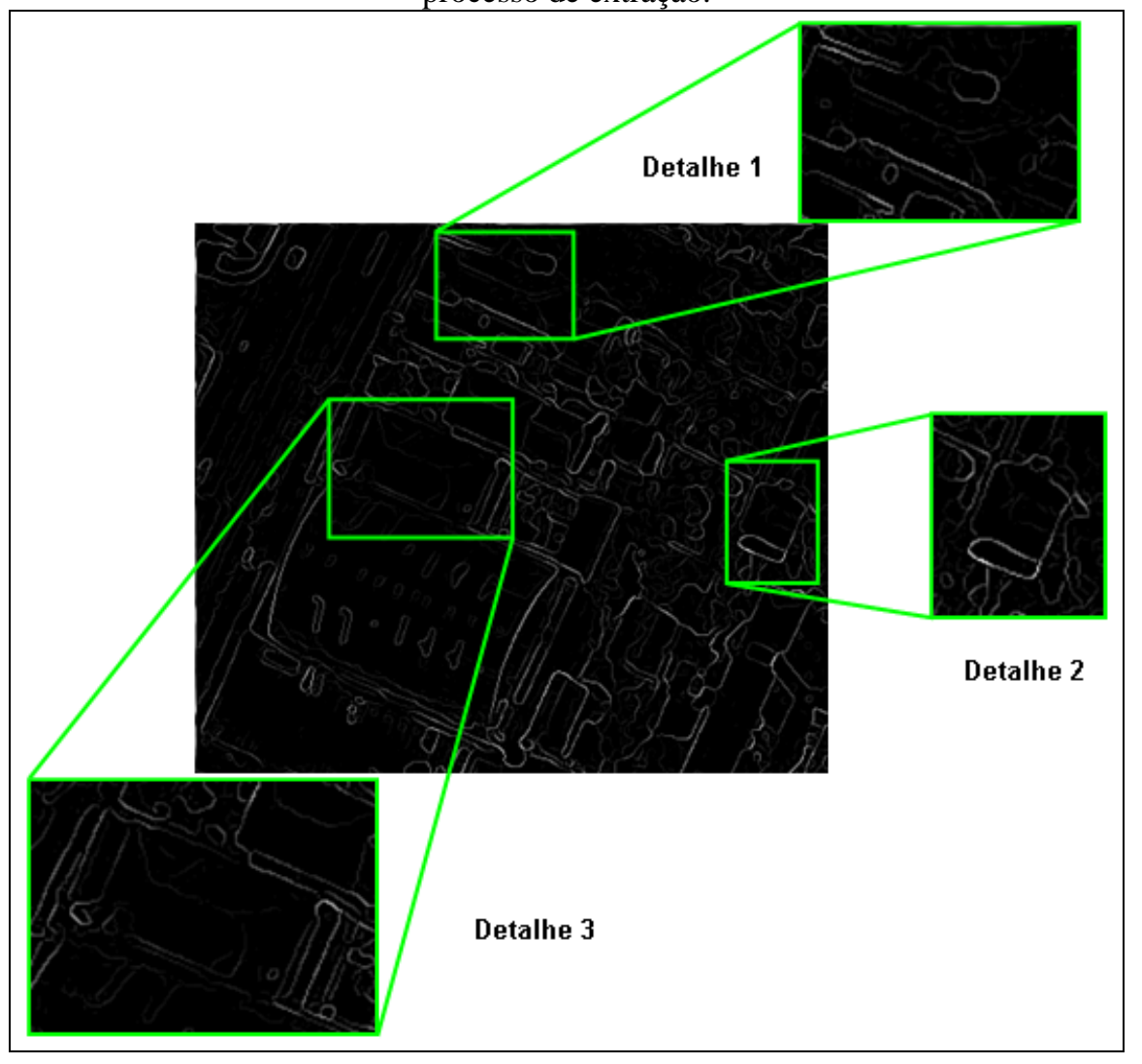

\section{CONCLUSÕES}

A partir da inspeção visual e dos resultados obtidos na análise numérica dos experimentos realizados é possível concluir que o método funciona adequadamente na extração de diferentes contornos de telhado de edifícios a partir de imagens digitais. Além disso, também foi possível verificar que snakes são modelos bastante flexíveis para modelagem de formas e extração de feições, uma vez que permitem a inclusão de diferentes informações a respeito dos objetos de interesse através da 
modelagem da função de energia externa da curva. Por outro lado, os modelos resultantes são sensíveis à qualidade dos dados de entrada. Em outras palavras, isto significa que as feições a serem extraídas devem estar bem definidas na imagem quando um modelo baseado em snakes é utilizado, conforme foi possível observar em alguns dos resultados obtidos nos experimentos realizados. Outro fator que interfere negativamente no desempenho do método e na qualidade dos resultados obtidos é a projeção de sombras nas regiões vizinhas aos contornos de telhado, conforme pode ser observado em alguns dos resultados obtidos nos experimentos realizados.

A principal direção para trabalhos futuros envolve o desenvolvimento de estratégias para evitar o efeito indesejado das sombras adjacentes aos edifícios. Quando está bem próxima e aproximadamente paralela ao edifício em análise, normalmente é difícil evitar que o algoritmo se confunda. Na presença de uma sombra com estas características, o vetor gradiente para pontos de borda do edifício aponta para o interior do telhado, enquanto que, para pontos de borda da sombra, aponta aproximadamente no sentido contrário. Essa propriedade pode ser explorada para modificar o termo de energia de borda, a fim de que sua energia seja atenuada. Uma possibilidade mais sofisticada é reformular a função de energia proposta no espaço-objeto, a fim de que ela possa incorporar também restrições específicas desse espaço, como a de desnível entre telhados e o terreno.

\section{AGRADECIMENTOS}

Este trabalho é parte de uma tese de doutorado em desenvolvimento junto ao Programa de Pós-Graduação em Ciências Cartográficas da Faculdade de Ciências e Tecnologia da UNESP - Universidade Estadual Paulista, com o apoio financeiro da FAPESP - Fundação de Amparo à Pesquisa do Estado de São Paulo.

\section{REFERÊNCIAS BIBLIOGRÁFICAS}

AGOURIS, P. et al. Dynamic node distribution in adaptive snakes for road extraction. $14^{\text {th }}$ Annual Vision Interface Conference. Proceedings, p. 134-140, Ottawa, 2001.

BALLARD, D.; BROWN, C. M. Computer vision. New Jersey: Prentice Hall, Inc., Englewood Cliffs, 1982, 523 p.

DAL POZ, A. P.; GALLIS, R. B. A.; SILVA, J. F. C. Three-dimensional semiautomatic road extraction from a high-resolution aerial image by dynamicprogramming optimization in the object space. IEEE Geoscience and Remote Sensing Letters, v. 7, n. 4, p. 796-800, 2010.

DAL POZ, A. P.; VALE, G. M. Dynamic programming approach for semiautomated road extraction from medium- and high-resolution images. ISPRS Archives, v. 34, n. 3/W8, p. 87-91, Munich, 2003.

GUERCKE, R.; GÖTZELMANN, T; BRENNER, C.; SESTER, M. Aggregation of LoD 1 building models as an optimization problem. ISPRS Journal of Photogrammetry and Remote Sensing, v. 66, n. 2, p. 209-222, 2011.

Bol. Ciênc. Geod., sec. Artigos, Curitiba, v. 17, nº 3, p.340-360, jul-set, 2011 
GRUEN, A.; LI, H. Road extraction from aerial and satellite images by dynamic programming. ISPRS Journal of Photogrammetry and Remote Sensing, v. 50, n. 4, p. 11-20, 1995.

GUO, T.; YASUOKA, Y. Snake-based approach for building extraction from highresolution satellite images and height data in urban areas. 2003. Accessed 30/06/10. http://www.gisdevelopment.net/aars/acrs/2002/vhr/018.pdf.

HARRIS, C.; STEPHENS, M. A combined corner and edge detector. Alvey Vision Conferece, University of Manchester, Proceedings, p. 147-151, 1988.

KASS, M.; WITKIN, A.; TERZOPOULOUS, D. Snakes: Active contour models. International Journal of Computer Vision, v. 1, n. 4, p. 321-331, 1988.

LAFARGE, F. et al. An Automatic Building Reconstruction Method: A Structural Approach using High Resolution Satellite Images. IEEE International Conference on Image Processing 2006, Proceedings, p. 1205-1208, 2006.

LEE, D. S.; SHAN, J.; BETTEL, J. S. Class-Guided Building Extraction from IKONOS Imagery. Photogrammetric Engineering \& Remote Sensing, v. 69, n. 2, p. 143-150, 2003.

LIU, Z. J. et al. Building extraction from high resolution imagery based on multiscale object oriented classification and probabilistic Hough transform. Geoscience and Remote Sensing Symposium 2005. Proceedings, vol. 4, p. 22502253, 2005.

LIU, Z. J. et al. Building extraction from high resolution satellite imagery based on multi-scale image segmentation and model matching. International Workshop on Earth Observation and Remote Sensing Applications, 2008. Proceedings, p. 1-7, 2008.

ORIOT, H. Statistical snakes for building extraction from stereoscopic aerial images. ISPRS Archives, Vol. XXXIV, Part 3/W8, Munich, 17.-19. Sept. 2003, p. 65-70.

PENG, J.; ZHANG, D.; LIU, Y. An improved snakes model for building detection from urban aerial images. Pattern Recognition Letters, v. 26, p. 587-595, 2005.

POULLIS, C.; YOU, S. Delineation and geometric modeling of road networks. ISPRS Journal of Photogrammtry and Remote Sensing, v. 65, p. 165-181, 2010.

RÜTHER, H.; MARTINE, H. M.; MTALO, E. G. Application of snakes and dynamic programming optimisation technique in modeling of buildings in informal settlement areas. ISPRS Journal of Photogrammetry and Remote Sensing, v. 56, p. 269-282, 2002.

SOHN, H.-G. et al. 3-D building extraction using IKONOS multispectral images. Geoscience and Remote Sensing Symposium 2005. Proceedings, vol. 2, p. 14321434, 2005.

XIONG, Z.; ZHANG, Y. Automatic 3D Building Extraction from Stereo IKONOS Images. Geoscience and Remote Sensing Symposium 2006. Proceedings, p. 3283-3286, 2006.

XU, C.; PRINCE, J. L. Generalized gradient vector flow external forces for active contours. Signal Processing, v. 71, 1998, p. 131-139. 
XU, C.; PRINCE, J. L. Snakes, shapes, and gradient vector flow. IEEE Transactions on Image Processing. v. 7, n. 3, p. 359-369. 1998.

(Recebido em abril de 2011. Aceito em julho de 2.11) 